\title{
Advanced Methods for Radiolabeling Multimodality Nanomedicines for SPECT/MRI and PET/MRI
}

\author{
Jennifer Lamb and Jason P. Holland \\ Department of Chemistry, University of Zurich, Zurich, Switzerland
}

The advent of hybrid cameras that combine MRI with either SPECT or PET has stimulated growing interest in developing multimodality imaging probes. Countless options are available for fusing magnetically active species with positron- or $\gamma$-ray-emitting radionuclides. The initial problem is one of choice: which chemical systems are a suitable basis for developing hybrid imaging agents? Any attempt to answer this question must also address how the physical, chemical, and biologic properties of a unified imaging agent can be tailored to ensure that optimum specificity and contrast are achieved simultaneously for both imaging modalities. Nanoparticles have emerged as attractive platforms for building multimodality radiotracers for SPECT/MRI and PET/MRI. A wide variety of nanoparticle constructs have been utilized as radiotracers, but irrespective of the particle class, radiolabeling remains a key step. Classic methods for radiolabeling nanoparticles involve functionalization of the particle surface, core, or coating. These modifications typically rely on using traditional metal ion chelate or prosthetic group chemistries. Though seemingly innocuous, appending nanoparticles with these radiolabeling handles can have dramatic effects on important properties such as particle size, charge, and solubility. In turn, alterations in the chemical and physical properties of the nanoparticle often have a negative impact on their pharmacologic profile. A central challenge in radiolabeling nanoparticles is to identify alternative chemical methods that facilitate the introduction of a radioactive nuclide without detrimental effects on the pharmacokinetic and toxicologic properties of the construct. Efforts to solve this challenge have generated a range of innovative chelate-free radiolabeling methods that exploit intrinsic chemical features of nanoparticles. Here, the chemistry of 9 mechanistically distinct methods for radiolabeling nanoparticles is presented. This discourse illustrates the evolution of nanoparticle radiochemistry from classic approaches to modern chelate-free or intrinsic methods.

Key Words: nanoparticles; intrinsic radiolabeling; chelate-free; chemisorption; doping; isotopic exchange; cation exchange; PET/MRI

J Nucl Med 2018; 59:382-389

DOI: 10.2967/jnumed.116.187419

$\mathbf{N}$ anomedicine is the application of nanotechnology to diagnose or treat disease (1). In the fields of radiochemistry and nuclear medicine, nanoparticles are gaining prominence as platforms for designing hybrid imaging and therapeutic agents (2-5). In

Received Aug. 30, 2017; revision accepted Sep. 30, 2017.

For correspondence or reprints contact: Jason P. Holland, Department of Chemistry, University of Zurich, Winterthurerstrasse 190, Zurich, 8057, Switzerland.

E-mail: jason.holland@chem.uzh.ch

Published online Oct. 12, 2017.

COPYRIGHT (C 2018 by the Society of Nuclear Medicine and Molecular Imaging. particular, nanoparticle-based radiotracers show promise as multimodality probes for SPECT/MRI and PET/MRI. The attention attracted by nanoparticles can be ascribed to their unique physical and chemical characteristics. Enhanced rigidity, controlled shape and size, discrete charge and electromagnetic properties, high surface area-to-volume ratios, variable porosity, resistance to metabolism in vivo, and tunable chemical reactivity at the surface, on coatings, and inside the particle core are just some of the features that demarcate nanoparticles as highly versatile scaffolds.

Despite their potential, there are many questions and challenges that must be addressed before nanoparticle-based imaging agents can cross the divide from preclinical to clinical applications. From a chemical standpoint, nanoparticle-based agents are highly complex. Average formulations contain particles with varying chemical compositions that include a range of sizes, variable drugloading densities, and differential presentations of targeting vectors in terms of their number per particle as well as their spatial location and 3-dimensional orientation. For this reason, most measurements made using nanoparticles rely on determination of the average properties of a bulk sample. Such inherent variability is a potential problem for ensuring batch-to-batch reproducibility and can induce differences in the biological properties of nanoparticles. Parameters such as the pharmacokinetic profile, cytotoxicity, target affinity, and specificity must remain consistent between formulations (6). It is easy to appreciate that although having access to such a wide range of physical and chemical parameters is beneficial from a design perspective, controlling each part of a nanoparticle system to meet stringent current goodmanufacturing-practice regulations is a formidable task.

When one is considering the development of a single molecular imaging probe that can be used to acquire nuclear and MR images simultaneously, several important design criteria must be incorporated into the final construct. For instance, a successful radiolabeled SPECT/MRI or PET/MRI probe should display high chemical, radiochemical, and metabolic stability; low toxicity; and a favorable pharmacokinetic profile with rapid accumulation in the target regions matched by excretion from background organs (7). A crucial question that has yet to be answered in detail is how one molecule can fulfil the usual chemical and physical requirements of nuclear and magnetic imaging probes without compromising on target specificity or image contrast. This problem is highlighted by the acute difference in the normal administered concentrations between standard PET radiotracers and MRI contrast agents. Clinicalgrade ${ }^{18} \mathrm{~F}$-labeled PET radiotracers typically have specific activities of around $185 \mathrm{GBq} / \mu \mathrm{mol}(5 \mathrm{Ci} / \mu \mathrm{mol})$, with administered radioactive doses in humans of around 370-740 MBq (10-20 mCi) (8). For an average man $(\sim 75 \mathrm{~kg})$, the administered dose of radiotracer equates to around $2-4 \mathrm{nmol}(\sim 25-50 \mathrm{pmol} / \mathrm{kg}$ of body weight). Typical gadolinium-based MRI contrast agents are administered at relatively high doses of up to $0.2 \mathrm{mmol}$ of gadolinium per kilogram 
of body weight (9). These administered doses (and sensitivities) represent a staggering difference from PET probes of about 7 orders of magnitude. How can a single, multimodality radiotracer for PET/ MRI reconcile this difference in concentration while maintaining high contrast in MRI and avoiding target saturation, which would reduce PET signal uptake and specificity? For a given combination of biologic target and imaging probe, does a concentration range exist that would be an acceptable compromise for simultaneous PET/MRI (or SPECT/MRI)? At present, these questions have not been addressed adequately. Most reports on multimodality imaging agents have tended to decouple the nuclear and imaging components by showing that a single species (usually nanoparticles) administered at different doses can be applied for sequential SPECT/MRI and PET/MRI. Recent work by Zhao et al. is a notable exception (10). SPECT/MRI studies showed that a single dose of ${ }^{125}$ I-radiolabeled human heavy-chain ferritin nanocages could image HT-29 tumors at a dose of $18.5 \mathrm{MBq}(500 \mu \mathrm{Ci})$ of ${ }^{125} \mathrm{I}$ and $11.2 \mu \mathrm{g}$ of iron. However, it remains uncertain if a similar approach would be generally applicable to more diverse imaging biomarkers of low abundance.

Returning to the chemistry of nanoparticles, a practical consideration is that the radiolabeling step should be easy and reproducible. Ideally, radiolabeling should be performed in the final step of production, and addition of the radionuclide should not alter (or should have known minimal effects on) the physical, chemical, and biologic properties of the nanoparticle. These demanding requirements have led researchers to develop several innovative solutions for producing radiolabeled nanoparticles. In the following sections, the chemistry and application of 9 conceptually distinct methods for radiolabeling different types of nanoparticles are explored. Classic radiolabeling methods based on modification of nanoparticles using metal ion chelation and prosthetic group chemistry are briefly introduced. The focus of this review is to illustrate how intrinsic chemical properties of nanoparticles are being harnessed by state-ofthe-art chelate-free radiolabeling methods to produce multimodality imaging agents (11-13).

\section{CLASSIC RADIOLABELING METHODS}

\section{Modifications to Particle Surface}

Traditional approaches for radiolabeling nanoparticles rely on well-established radiochemical methods using ${ }^{18} \mathrm{~F}$-based prosthetic groups or radiometal ion chelation. Here, nanoparticles are typically coated with surface reactive groups that serve a dual purpose. First, they allow covalent attachment of radionuclides via prosthetic groups or multidentate chelates. Second, they provide thermodynamically, kinetically, and metabolically stable anchors ensuring that the radionuclide remains associated with the nanoparticle in vivo. Three common examples of surface anchoring

\section{NOTEWORTHY}

- Effective design of multimodality nanomedicines for SPECT/ MRI and PET/MRI requires new radiochemical methods to overcome limitations imposed by conventional prosthetic group and chelate-based chemistries.

- Chelate-free methods exploit intrinsic chemical properties of nanoparticles to effect facile and efficient radiolabeling.

- Mechanistic principles underpinning 7 distinct chelate-free radiolabeled methods are presented. include thiol-mediated binding to gold nanoparticles (14-16), silylation of surface hydroxyls (17), and bisphosphonate binding $(18,19)$ to iron oxide particles (Fig. 1).

In an archetypical example, Guerrero et al. (15) produced ${ }^{18} \mathrm{~F}$ labeled gold nanoparticles. They were prefunctionalized with a cysteine-lysine diamino acid via covalent bonding of the thiolate to the gold surface. Radiolabeling was accomplished by attaching the ${ }^{18} \mathrm{~F}$-radiolabeled prosthetic group, $N$-succinimidyl-4- ${ }^{18} \mathrm{~F}$ fluorobenzoate, via amide bond formation on the lysine sidechain. Rojas et al. (17) applied a similar strategy but used 3-(aminopropyl)triethoxysilane to decorate the surface of $\mathrm{CeO}_{2}$ nanoparticles with a primary amine that was subsequently coupled to $N$-succinimidyl-4- ${ }^{18} \mathrm{~F}$-fluorobenzoate. A major drawback of this approach is that multiple radiolabeling steps are required, limiting overall radiochemical yields and specific activities. Others have sought to overcome these issues by developing a cysteamine derivative of ${ }^{18} \mathrm{~F}-\mathrm{FDG}$ that binds to gold nanoparticles (14). Zhu et al. (16) also reported an interesting approach to make ${ }^{18} \mathrm{~F}-$ radiolabeled PEGylated gold nanoparticles that relied on $\mathrm{Si}^{18} \mathrm{~F}$ bond formation. The thiol (4-(di-tert-butyl- ${ }^{18} \mathrm{~F}$-fluorosilanyl)benzenethiol) was produced via ${ }^{18} \mathrm{~F} /{ }^{19} \mathrm{~F}$ isotopic exchange and was subsequently conjugated to maleimido-gold nanoparticles, giving high radiochemical yields $(60 \%-87 \%)$. Although this prosthetic-group approach solves some of the issues associated with low radiochemical yields, it is not clear how much of the thiol reacts with the maleimido group and how much reacts directly with the gold nanoparticle surface.

In 2011, Rosales et al. used bisphosphonate chemistry to generate SPECT/MRI and PET/MRI radiotracers based on conjugation of superparamagnetic iron oxide nanoparticles (SPIONs) with either ${ }^{99 \mathrm{~m} T c}$ or ${ }^{64} \mathrm{Cu}(18,19)$. Bisphosphonates display well-established affinity for several different classes of inorganic surfaces, including various metal oxides, and hydroxyapatite crystals found in bone. Both studies followed a similar approach in which multidentate chelates, chosen for their selectivity toward a particular radiometal ion, were functionalized with a bisphosphonate group. ${ }^{64} \mathrm{Cu}^{2+}$ bis(di-thiocarbamatebisphosphonate) and ${ }^{99 \mathrm{~m}} \mathrm{Tc}$-dipicolylamine (DPA)-alendronate were conjugated to ferumoxide (Endorem [Guerbet] or Feridex [Advanced Magnetics, Inc.])—dextran-coated magnetite $\left(\mathrm{Fe}_{3} \mathrm{O}_{4}\right)$ nanoparticles $(120-180 \mathrm{~nm}$ in diameter) approved for T2- or T2*-weighted contrast-enhanced MRI (20). Detailed stability studies in vitro and multimodality imaging in vivo confirmed the viability of bisphosphonate chemistry for radiolabeling metal oxide nanoparticles (21).

\section{Modifications to Particle Coating}

The second fundamental approach to radiolabeled nanoparticles involves modification to the particle coating. Radiolabeling reactions on particle coatings involve prosthetic group or chelate-based chemistries similar to those described above. The key difference is that surface-anchoring groups are not required for radiolabeling of coatings. Instead, the radioactive group is introduced to the coating via covalent bond formation. Again, many examples have been reported, and we highlight just 2 representative approaches (Fig. 2).

In 2009, Devaraj et al. reported the synthesis and use of ${ }^{18} \mathrm{~F}$ radiolabeled cross-linked dextran iron oxide nanoparticles for PET/CT imaging (22). The innovative step in radiolabeling involved derivatization of the dextran coating with a reactive azide group. Subsequent copper-catalyzed azide-alkyne cycloaddition click reactions using a presynthesized ${ }^{18} \mathrm{~F}-\mathrm{PEG}_{3}$ alkyne reagent 


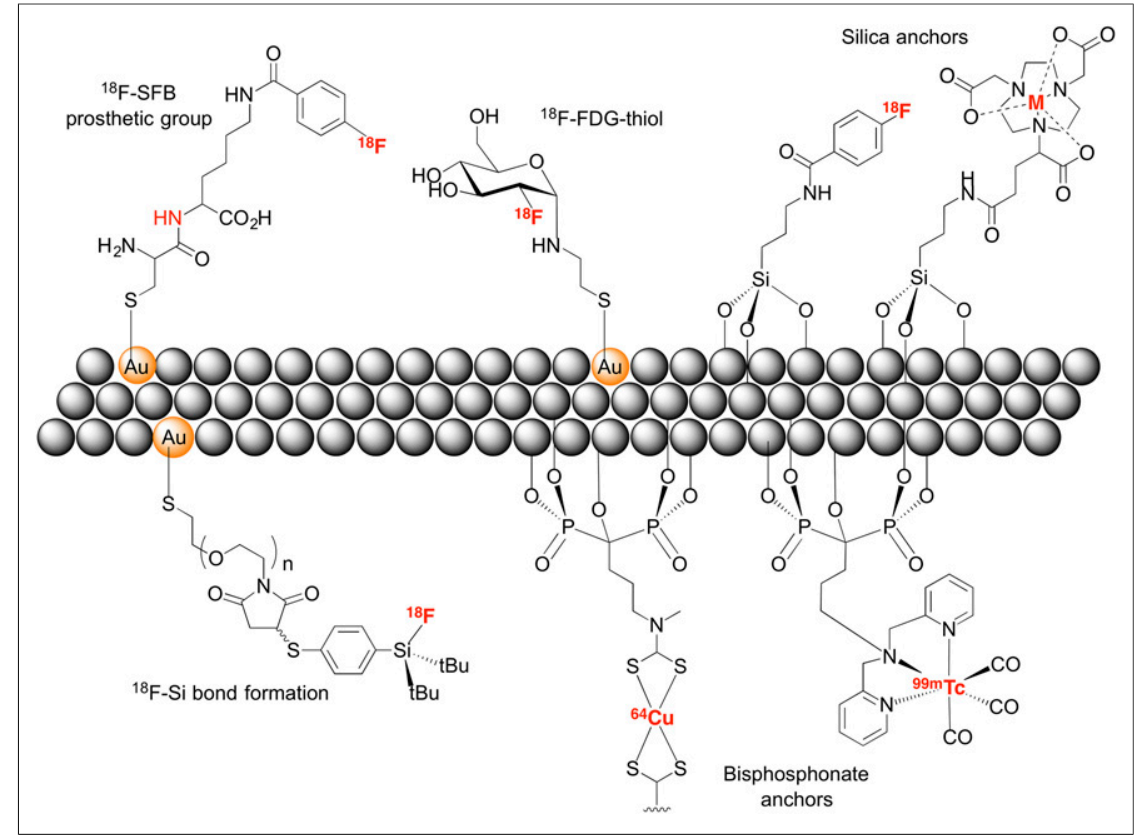

FIGURE 1. Some of the classic, surface-based methods that have been used to radiolabeled nanoparticles.

(produced in an average 57\% decay-corrected radiochemical yield) facilitated rapid ${ }^{18} \mathrm{~F}$ radiolabeling of azide-functionalized cross-linked dextran iron oxide nanoparticles in $40 \mathrm{~min}$ at $40^{\circ} \mathrm{C}$. As with most nanoparticle-based radiotracers, purification from the nonreacted ${ }^{18} \mathrm{~F}-\mathrm{PEG}_{3}$-alkyne was achieved using a simple filtration step to give the final ${ }^{18} \mathrm{~F}$-labeled cross-linked dextran iron oxide product in 58\% decaycorrected radiochemical yield and more than $99 \%$ radiochemical purity. This work showcases the potential of click chemistry for producing complex radiolabeled constructs in a simple, high-yielding, and biochemically orthogonal process.

Coating-based metal ion chelation was used by Thorek et al. to produce ${ }^{89} \mathrm{Zr}$ desferrioxamine (DFO)-labeled ferumoxytol (Feraheme; AMAG Pharmaceuticals, Inc.) for use in PET/MRI of sentinel lymph nodes (Fig. 2) (23). In a 3-step process, the carboxymethyl dextran coating of ferumoxytol was functionalized with 1,2-ethylenediamine followed by conjugation to DFO-pBn-SCN and subsequent radiolabeling with ${ }^{89} \mathrm{Zr}$-oxalate to produce ${ }^{89} \mathrm{Zr}$-DFO-ferumoxytol. PET/MRI in a transgenic mouse model of prostate cancer displaying elevated levels of MYC transcription factor activity showed localization of ${ }^{89} \mathrm{Zr}$-DFO-ferumoxytol in prostate-draining lymph nodes. Although this DFO-based method to radiolabel ferumoxytol with ${ }^{89} \mathrm{Zr}$ was recently superseded by a chelate-free approach (described below) (24), this study provides a convincing demonstration of the need for PET/MRI of deep-seated

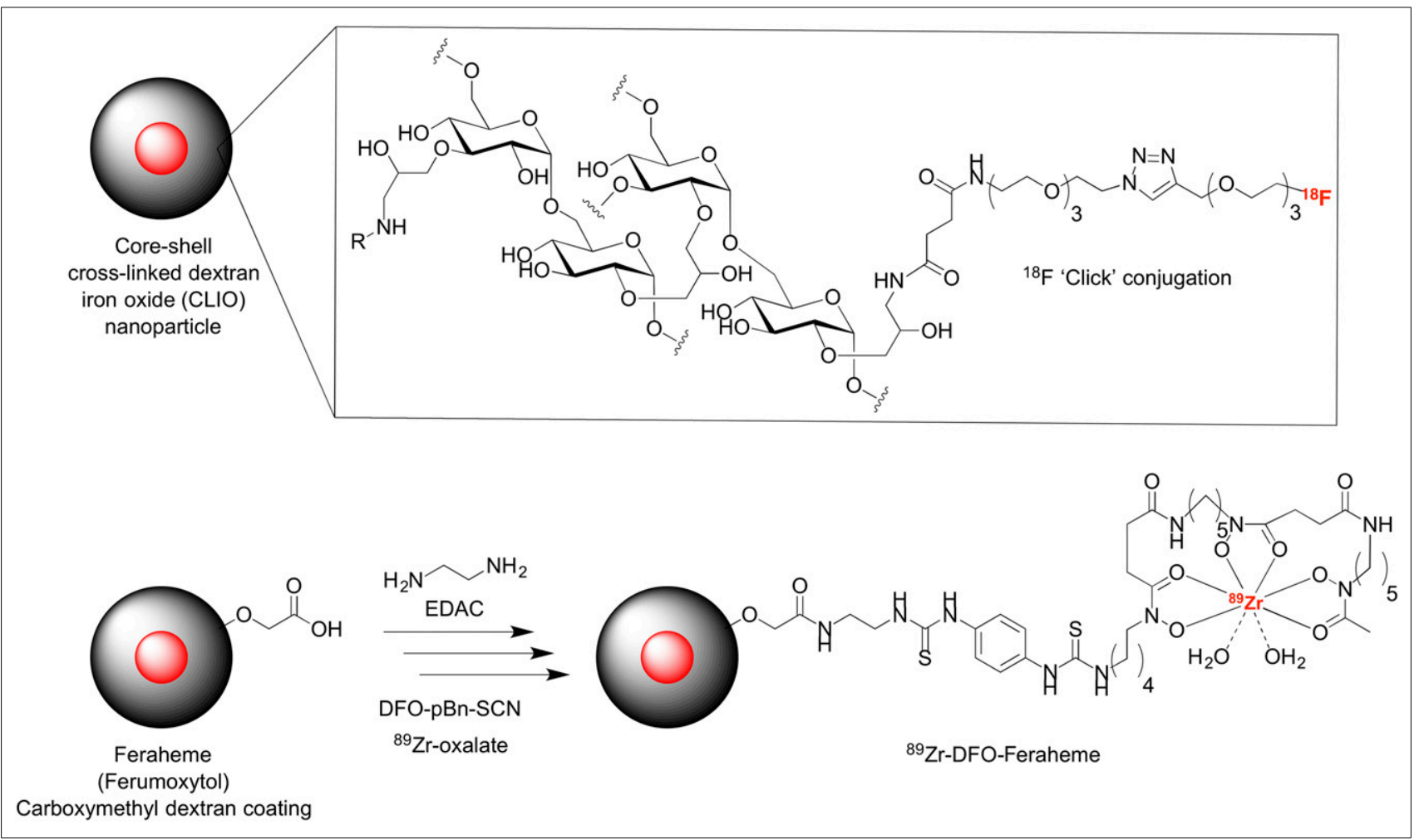

FIGURE 2. Two prominent examples of classic coating-based radiolabeling methods. 


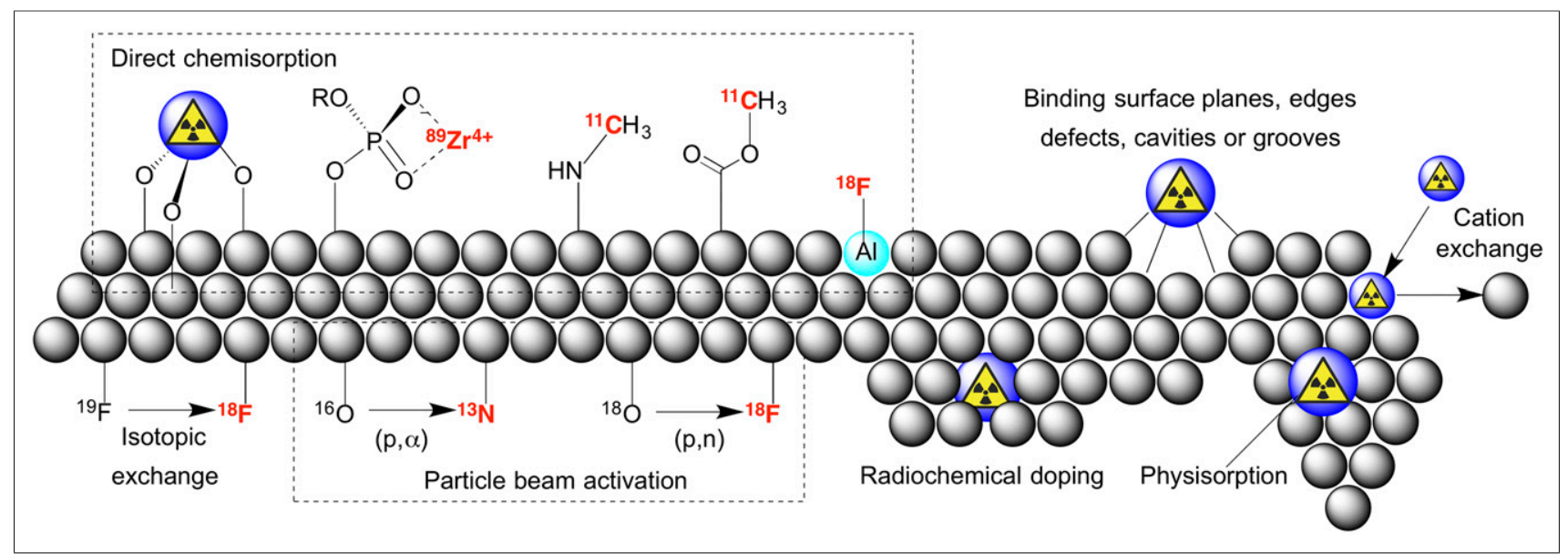

FIGURE 3. The diversity of emerging chelate-free methods that are being developed for radiolabeling different nanoparticles.

tissues that are difficult to identify and characterize using singlemodality cameras.

\section{NONCLASSIC RADIOLABELING METHODS}

A disadvantage of classic radiolabeling methods is that the introduction of a prosthetic group or metal ion chelate can have adverse effects on the pharmacokinetic and toxicity profiles of the nanoparticle $(6,7)$. For this reason, efforts have been made to identify alternative methods for radiolabeling nanoparticles that avoid the use of often bulky, lipophilic prosthetic groups or chelates that alter surface charge (Fig. 3).

\section{Radiochemical Doping}

This nonclassic approach has previously been described as the addition of hot-plus-cold precursors $(11,25)$. A scientifically more accurate description is radiochemical doping-defined here as a process in which radiolabeled nanoparticles (or more general composites) are made via the addition of small amounts of a radionuclide during particle fabrication (Fig. 4). The method draws on well-established protocols that have their origins in the FajansPaneth-Hahn law of radioactive coprecipitation (as described in Otto Hahn's 1936 work, Applied Radiochemistry). The law governs how a radioactive trace element coprecipitates in the presence of a larger amount of carrier material. If experimental conditions such as solubility, concentrations of precipitants, ionic strength, and counter ion identity are controlled, then it is possible to generate so-called mixed crystals in which the trace radionuclide is

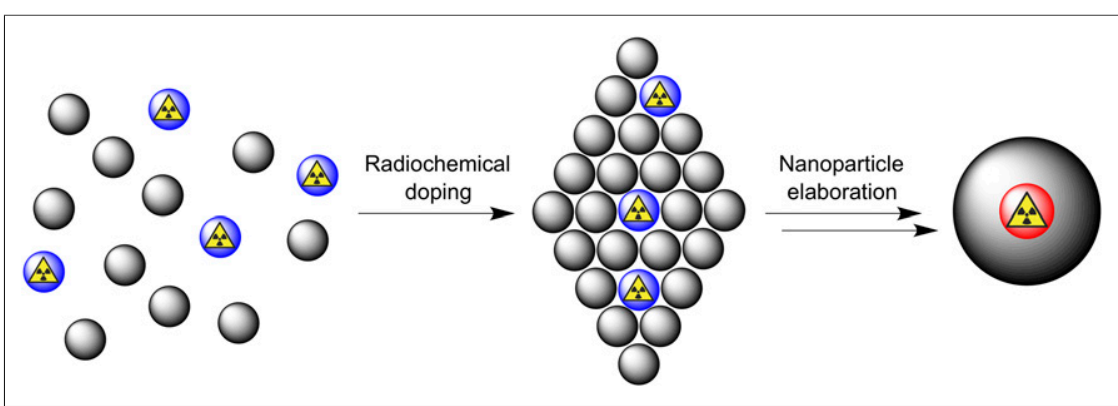

FIGURE 4. Concept of radiochemical doping during nanoparticle fabrication. incorporated into the crystal structure of the particulate. Notably, the law also states that if particles acquire a surface charge that is opposite to the charge on the trace element, then coprecipitation of the radioactivity will depend strongly on the conditions used, with the tracer likely to become chemically or physically adsorbed onto the particle surface (described in the sections on chemisorption and physisorption).

Numerous reports using coprecipitation have proven that radiochemical doping is an effective means for generating a wide range of radiolabeled nanoparticles involving different core materials and radionuclides. Radiochemical doping has been achieved using ${ }^{64} \mathrm{Cu}(26-30),{ }^{65} \mathrm{Zn}(31),{ }^{68} \mathrm{Ga}(32),{ }^{109} \mathrm{Cd}(33),{ }^{111} \mathrm{In}(31,34)$, ${ }^{141} \mathrm{Ce}(31),{ }^{153} \mathrm{Sm}(35)$, and ${ }^{198} \mathrm{Au}(36,37)$ radionuclides to produce multimodality particles.

In a state-of-the-art example of homoradionuclide doping, Black et al. produced a range of shape- and size-controlled PEGylated gold nanostructures loaded with ${ }^{198} \mathrm{Au}$ (half-life, $2.69 \mathrm{~d} ; \beta^{-}, 100 \%$ ). All nanoparticles were of a similar size, but the authors produced different nanostructures, including nanospheres, nanodisks, nanorods, and cubic nanocages. A combination of ex vivo biodistribution data and in vivo SPECT coupled with Cerenkov luminescent imaging and $\mathrm{CT}$ was used to measure tumor localization in a mouse model bearing murine EMT6 breast carcinoma. Remarkably, pharmacokinetic profiles and intratumoral studies showed that radiotracer distribution was heavily dependent on the particle shape. The ${ }^{198} \mathrm{Au}-$ nanospheres showed the longest blood pool residence time and the highest uptake in tumors, reaching $23.2 \%$ injected dose per gram at $24 \mathrm{~h}$. Understanding how the physical shape of nanoparticles influences their behavior in vivo is an emerging frontier in nanoscience that will likely have an impact on the future design of multimodality radiotracers (37).

It is important to note that, whereas radiochemical doping is a fairly general synthetic strategy, not all metal ions and metal-based nanoparticles are compatible. For example, Zeng et al. studied the properties of ${ }^{111}$ In- and ${ }^{57} \mathrm{Co}$-doped PEGylated $\mathrm{Fe}_{3} \mathrm{O}_{4}$ nanoparticles (34). Radiochemical stability measurements found that ${ }^{111} \mathrm{In}$ doped nanoparticles remained intact at $\mathrm{pH}$ 2. However, $21.0 \%$ of the radioactivity 
was leached from the ${ }^{57} \mathrm{Co}$-doped nanoparticles after dialysis for 24 $\mathrm{h}$ in water. The difference in the dopant leaching was attributed to the different solubility products of the corresponding hydroxides ( 2 $\times 10^{-16}$ for $\mathrm{Co}(\mathrm{OH})_{2}$ and $1.3 \times 10^{-37}$ for $\left.\mathrm{In}(\mathrm{OH})_{3}\right)$. This work provides important lessons that dopant-nanoparticle chemistry must be matched and that careful stability measurements should always be performed before conducting biologic assays in vitro and in vivo.

\section{Physisorption}

Although doping involves incorporation of the radionuclide into the core of the nanoparticle crystal, most other nonclassic nanoparticle radiolabeling methods make use of surface-based chemistry. Physical adsorption (physisorption) is a process by which small molecules or ions interact and associate with a molecular surface via electrostatic attraction or van der Waals interactions. The key feature of physisorption is that no discrete covalent or dative covalent bond is made between the species binding to the particle and the surface itself. Solid nanoparticles dispersed as a colloid in solution typically acquire a surface charge known as the electric double-layer potential (Fig. 5). This charged layer is often characterized by the measured $\zeta$-potential (in units of $\mathrm{mV}$ ), with higher net charges (either positive or negative) correlating with increased stability of the colloid in solution and lower net charges favoring coagulation or flocculation. The $\zeta$-potential also affects absorption, distribution, metabolism, excretion, and toxicologic properties of nanoparticles $(39,40)$.

From a radiolabeling perspective, species (ions) that acquire a charge opposite that of the nanoparticle surface can become immobilized in the stationary layer between the particle surface and the dispersed medium. To the best of our knowledge, there are no specific reports of radiolabeling of nanoparticles via a physisorption process. However, the absence of data likely reflects our limited mechanistic knowledge on the interactions between radiometal ions and particle surfaces. Many nanoparticle-based

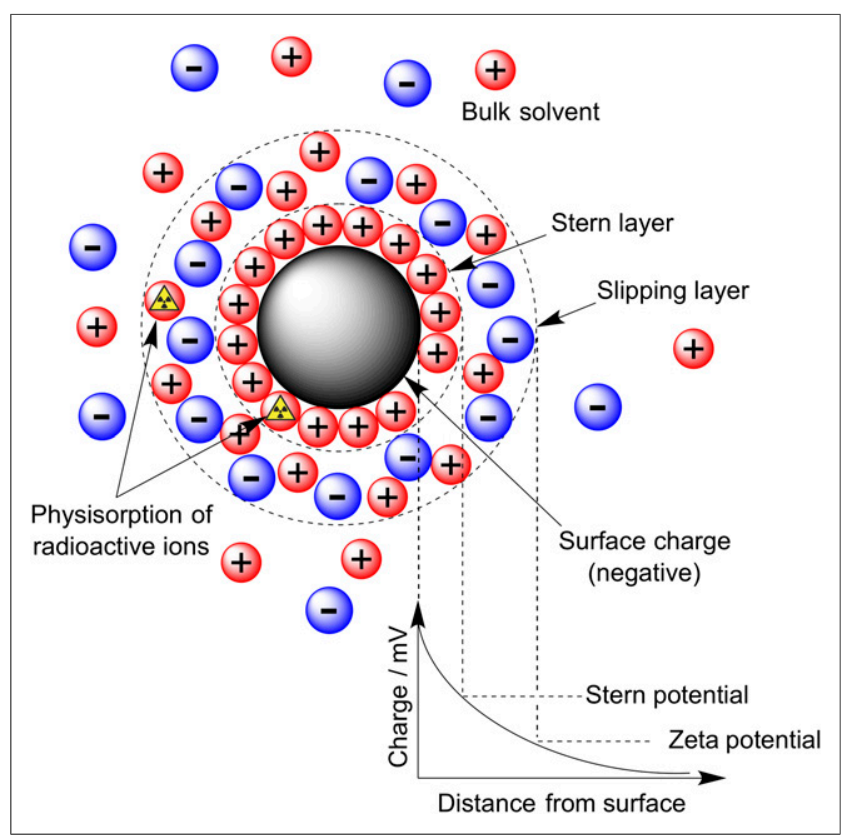

FIGURE 5. Distribution of charged ions around surface of nanoparticle in solution. systems acquire a negative $\zeta$-potential, and hence, it is intuitive that positively charged metal cation species can potentially form a tight ion pair with the surface and become trapped electrostatically in the immobile region. Further studies are required to elucidate if physisorption is a viable method for radiolabeling nanoparticles.

\section{Direct Chemisorption}

Arguably the most versatile and promising new method for radiolabeling a broad scope of nanoparticles involves direct chemical bond formation between the radionuclide and the particle surface (Fig. 3). In surface chemistry, this mechanism is called chemisorption. The approach was pioneered by the group of Weibo Cai et al. $(41-48)$. The concept has recently been generalized, first by our group (24) for metal ions from across the periodic table and subsequently by others for different classes of nanoparticle (49-52).

In 2013, Chen et al. reported an intriguing study in which the well-known affinity of $\mathrm{As}^{3+}$ and $\mathrm{As}^{5+}$ ions for the surface of magnetite $\left(\mathrm{Fe}_{3} \mathrm{O}_{4}\right)$ was exploited to develop a novel chelate-free approach for producing *As-SPIONs (where $*=71,72,74,76$ ) as potential PET/MRI agents (41). The high affinity of arsenic ions for $\mathrm{Fe}_{3} \mathrm{O}_{4}$ has been attributed to direct chemisorption in which $\mathrm{As}^{3+} \mathrm{O}_{3}$ trigonal pyramids or $\mathrm{As}^{5+} \mathrm{O}_{4}$ tetrahedra occupy vacant $\mathrm{FeO}_{4}$ tetrahedral sites on the octahedrally terminated $\{111\}$ surface of the magnetite nanoparticles. Control studies using citratecapped copper sulfide nanoparticles or SPIONs coated with dense $\mathrm{SiO}_{2}$ showed no appreciable radiolabeling, confirming that the adsorption process involved specific chemical interactions with the magnetite surface. Further studies in mice demonstrated that the PEGylated *As-SPION compounds were suitable radiotracers for mapping lymph-node drainage with PET/MRI. It is noteworthy that before the development of this chelate-free method, chemical options for radiolabeling molecules with $* A s^{n+}$ were restricted to *As-thiolate chelation. Follow-up studies have found that direct chemisorption is a viable synthetic route for producing multimodality radiotracers using different radionuclides $\left({ }^{69} \mathrm{Ge},{ }^{64} \mathrm{Cu}\right.$, ${ }^{89} \mathrm{Zr}$, and recently ${ }^{45} \mathrm{Ti}$ ) and nanoparticles, including SPIONs, iron oxide-coated $\mathrm{MoS}_{2}$ nanosheets, mesoporous silica nanoparticles, $\mathrm{Gd}_{2} \mathrm{O}_{2} \mathrm{~S}$ :Eu nanophosphors, and nanographene.

In 2015, our group demonstrated the generality of chelate-free chemisorption radiolabeling of iron oxide-based nanoparticles (24). Nonradioactive induction-coupled plasma mass spectrometry studies found that chemisorption could be used to label ferumoxytol nanoparticles with different metal ions, including $p$-block $\mathrm{In}^{3+}$; first-row $d$-block $\mathrm{Mn}^{2+}, \mathrm{Co}^{2+}, \mathrm{Cu}^{2+}$, and $\mathrm{Zn}^{2+}$; secondrow $\mathrm{Zr}^{4+}$; and $f$-block lanthanide ions such as $\mathrm{Eu}^{3+}$ and $\mathrm{Tb}^{3+}$ (Fig. 6A). Radiolabeling experiments using ${ }^{64} \mathrm{CuCl}_{2},{ }^{111} \mathrm{InCl}_{3}$, and ${ }^{89} \mathrm{Zr}$-oxalate demonstrated that using the same reaction conditions, radiolabeled ${ }^{64} \mathrm{Cu}$-ferumoxytol, ${ }^{111} \mathrm{In}$-ferumoxytol, and ${ }^{89} \mathrm{Zr}$-ferumoxytol could be produced in $66 \% \pm 6 \%, 91 \% \pm 2 \%$, and more than $95 \%$ decay-corrected radiochemical yields, respectively. This is a remarkable result given the differences in charge, ionic radius, and chemical requirements of these metal ions. In vitro characterization experiments found that the ferumoxytol nanoparticles were physically and chemically unchanged after radiolabeling and that the radioactivity remained tightly bound to the particles even in the presence of standard chelate challenge or serum stability assays. In most instances, the precise nature and location of the metal ion binding to the particle is not known. However, electron paramagnetic resonance studies using natural ${ }^{63 / 65} \mathrm{Cu}$-ferumoxytol provided a strong indication that the metal ions are surface-bound and do not interact with the core crystal structure of the magnetite 


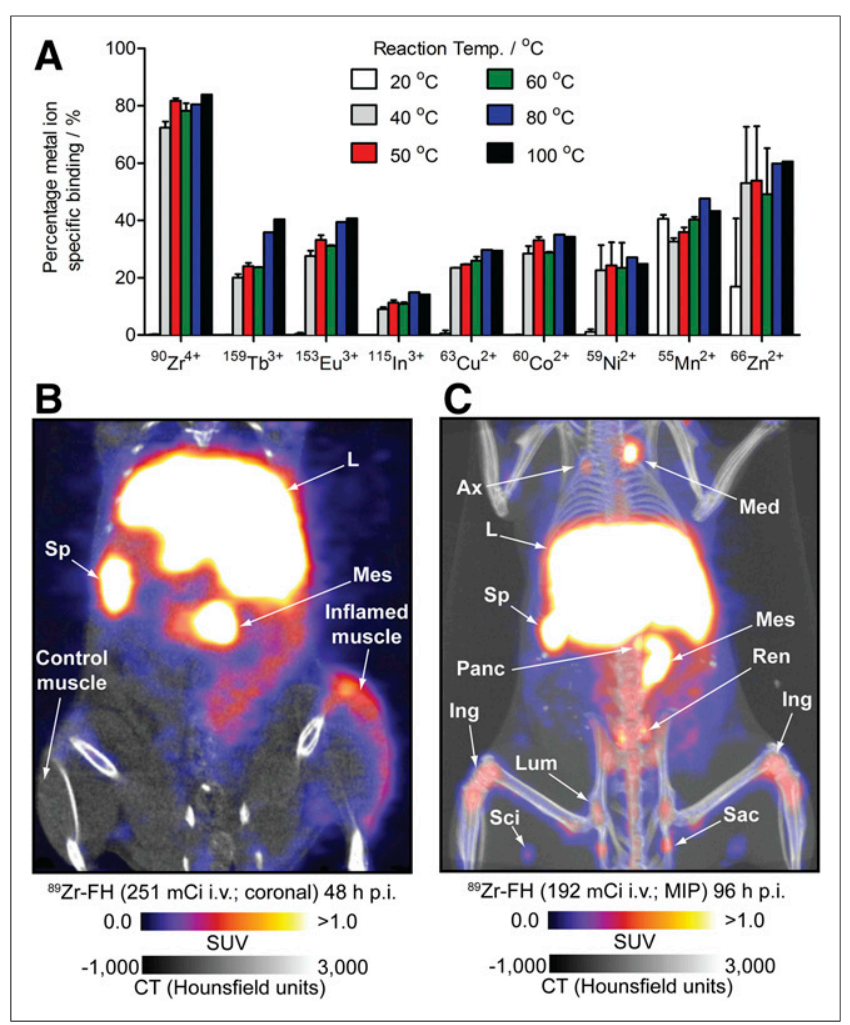

FIGURE 6. (A) Temperature-dependent induction-coupled plasma mass spectrometry data showing that metal ion salts from across periodic table can be used in chelate-free labeling. (B and C) PET/CT images showing uptake of ${ }^{89} \mathrm{Zr}$ - $\mathrm{FH}$ in acute-phase-response inflammation model $(B)$ and normal lymph nodes $(C)$. Ax = axillary lymph node; Ing = inguinal lymph node; $\mathrm{L}=$ liver; $\mathrm{Lum}=$ lumbar lymph node; Med = mediastinal lymph node; Mes = mesenteric lymph node; MIP = maximum-intensity projection; Panc = pancreas; Ren = kidney; Sac = sacral lymph node; $\mathrm{Sci}=$ sciatic lymph node; $\mathrm{Sp}=$ spleen .

SPIONs. Further studies are required to elucidate the precise nature of the chemical interactions between different metal ions and different surfaces. PET/MRI and CT experiments performed using ${ }^{89} \mathrm{Zr}$-ferumoxytol showed that the radiotracer is potentially useful for monitoring acute-phase-response inflammation (via accumulation in macrophages) or for detecting biochemical changes in the lymphatic system (Figs. 6B and 6C).

Subsequent work by Shaffer et al. also showed that direct chemisorption using ${ }^{64} \mathrm{Cu},{ }^{68} \mathrm{Ga},{ }^{89} \mathrm{Zr},{ }^{90} \mathrm{Y},{ }^{111} \mathrm{In}$, and ${ }^{177} \mathrm{Lu}$ was applicable for radiolabeling silica nanoparticles (49). Further examples of direct chemisorption include the interaction of ${ }^{89} \mathrm{Zr}^{4+}$ ions with phosphate groups of preformed liposomes $(53),{ }^{18} \mathrm{~F}$ radiolabeling of $\mathrm{Al}_{2} \mathrm{O}_{3}$-coated $\mathrm{MnFe}_{2} \mathrm{O}_{4}$ and $\mathrm{Fe}_{3} \mathrm{O}_{4}$ nanoparticles $(54),{ }^{18} \mathrm{~F}$ radiolabeling of rare-earth nanoparticles $(55,56)$, and ${ }^{11} \mathrm{C}$-methyl iodide radiolabeling of carboxylate- and amine-coated iron oxide nanoparticles (57). Collectively, reports on radiolabeling via direct chemisorption offer the general lessons that chelate-free methods typically do not disrupt the physical and biochemical properties of the nanoparticle and that the radionuclide-surface chemistry must be well matched for specific and stable bonding to occur.

\section{Isotope Exchange}

Substitution of a nonradioactive nuclide for a chemically equivalent radioactive nuclide is termed isotopic exchange. The method is most frequently encountered in the chemistry of ${ }^{19} \mathrm{~F} /{ }^{18} \mathrm{~F}$ exchange for radiolabeling small molecules. A limited number of studies that fall into this category of nonclassic radiolabeling of nanoparticles have been reported, but the approach is feasible. For example, Cui et al. (56) and Sun et al. (55) evaluated the properties of various ${ }^{18} \mathrm{~F}$-radiolabeled nanoparticles that incorporate $\mathrm{NaYF}_{4}$. At present, it remains unclear if the high affinity of ${ }^{18} \mathrm{~F}$-fluoride anions for $\mathrm{NaYF}_{4}$ surfaces involves a mechanistic addition (chemisorption) or a substitution (isotopic exchange). Nevertheless, as the diversity of nanoparticle-based radiotracers expands, simple isotopic exchange reactions may become more prevalent routes for radiolabeling.

\section{Cation Exchange}

Cation exchange is an established process in nanocrystal synthesis and materials science (58). Mechanistically, cation exchange is related to the isotopic exchange in that a substitution reaction occurs either on the surface or inside the core of the nanocrystal (Fig. 3). The key difference is that for cation exchange, the incoming radionuclide and the displaced cation can be chemically distinct. Perhaps the term heteronuclide exchange is a more appropriate one since it encompasses possible substitution of different atoms, irrespective of the charge. To the best of our knowledge, there have been only two reports on the use of cation exchange for radiolabeling nanoparticles.

Sun et al. produced ${ }^{64} \mathrm{Cu}$-radiolabeled $\mathrm{CdSe} / \mathrm{ZnS}$ quantum dots via $\mathrm{M}^{2+}$ cation exchange (59). The process was described as doping, but since the quantum dots were prefabricated before radiolabeling, the mechanism is more accurately classified as cation exchange or chemisorption. Interestingly, incorporation of ${ }^{64} \mathrm{Cu}$ radionuclides into the quantum dots induced the particles to auto-fluoresce after excitation from the emitted Cerenkov radiation. Subsequent measurements in mice bearing U87MG glioblastoma showed specific accumulation of ${ }^{64} \mathrm{Cu}$-quantum dots in the tumors and provided evidence that optical luminescence imaging can be fused with nuclear imaging in the form of PET/ Cerenkov luminescence imaging.

Separately, Sun et al. (60) reported that cation exchange could be used to radiolabel different mixed core-shell nanoparticles based on $\mathrm{NaLuF}_{4}: \mathrm{Yb}, \mathrm{Gd}, \mathrm{Tm}$ composites with ${ }^{153} \mathrm{Sm}$. This example lies at the borderline between cation exchange and radiochemical doping (35). The authors produced a composite nanoparticle consisting of $\mathrm{NaLuF}_{4}: \mathrm{Yb}, \mathrm{Tm}$ coated in a secondary shell of $\mathrm{NaGdF}_{4}\left({ }^{153} \mathrm{Sm}\right)$. A homogeneous solution of $\mathrm{GdCl}_{3}$ and ${ }^{153} \mathrm{SmCl}_{3}$ was added to preformed nanocrystals of $\mathrm{NaLuF}_{4}$ to form a radiolabeled coating using forcing conditions. Subsequent annealing at $300^{\circ} \mathrm{C}$ and redispersion in citrate produced cit- $\mathrm{NaLuF}_{4}: \mathrm{Yb}$, $\mathrm{Tm} @ \mathrm{NaGdF}_{4}\left({ }^{153} \mathrm{Sm}\right)$ nanocrystals. Although successful in the production of an optical/SPECT/CT imaging agent, such harsh radiolabeling conditions will limit potential biomedical applications of this method.

\section{Particle Beam or Reactor Activation}

An interesting concept toward radiolabeled nanoparticles is to presynthesize the nonradioactive variant and then use particle beam or reactor-based activation to transmute an atom in situ (Fig. 3) (5). Both neutron $(61,62)$ and proton $(63,64)$ activations have been reported to produce radiolabeled nanoparticles via ${ }^{18} \mathrm{O}(p, n){ }^{18} \mathrm{~F},{ }^{16} \mathrm{O}(p, \alpha){ }^{13} \mathrm{~N}$, and ${ }^{165} \mathrm{Ho}(n, \gamma){ }^{166} \mathrm{Ho}$ transmutation. For example, Munaweera et al. produced ${ }^{166} \mathrm{Ho}$-radiolabeled garnet nanoparticles loaded with various platinum-based chemotherapeutics (62). The magnetic ${ }^{166} \mathrm{Ho}-\mathrm{HolG}-\mathrm{Pt}$ nanoparticles were selectively 


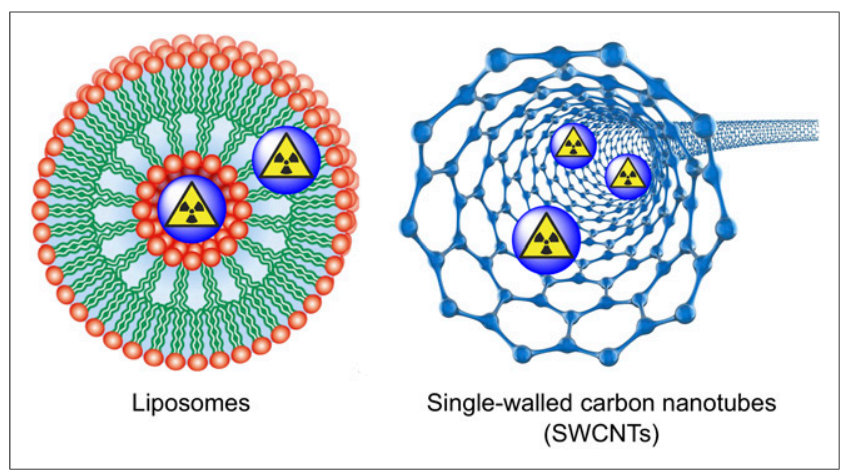

FIGURE 7. Cavity encapsulation of radiolabel inside liposomes and single-walled carbon nanotubes. (Underlying liposome figure reproduced under Creative Commons Attribution: Vladimir P. Shirinsky, http://eng.thesaurus.rusnano.com/wiki/article1075.)

delivered to a lung tumor model using an external magnetic field. Statistically significant decreases in tumor burden were noted for groups treated simultaneously with both active platinum complexes and the application of an external magnetic field versus controls. Although this inorganic system shows promise, lack of stability of organic materials in the particle beam will likely limit more widespread use of this method.

\section{Cavity Encapsulation}

The final method involves trapping a radioactive species inside the cavity of a nanoparticle. The process relies on a physical encapsulation that can also be described as a mechanical bond. Classic examples of systems that allow for drug encapsulation include liposomes (65) and single-walled carbon nanotubes (66) (Fig. 7). Although incorporation of radiometal ions into the aqueous-phase core of liposomes is a well-established method for producing radioscintigraphy and SPECT imaging agents using ${ }^{99 \mathrm{~m}} \mathrm{Tc}$, insertion of radionuclides into nanotubes is more challenging. In 2014, Cisneros et al. reported methods for the stable, simultaneous confinement of both $\mathrm{Gd}^{3+}$ ions and ${ }^{64} \mathrm{Cu}^{2+}$ ions into ultrashort single-walled carbon nanotubes (66). Radiolabeled nanotubes were stabilized using surfactants, and although some ${ }^{64} \mathrm{Cu}$ was removed by serum challenge, the constructs were sufficiently stable to allow evaluation of the pharmacokinetic profiles using PET/MRI. Since single-walled carbon nanotubes have a high loading capacity, and the $\mathrm{Gd}^{3+}$ ions and radionuclides contained within are shielded from the biologic environment, cavity encapsulation offers a potential solution for solving the concentration problem between PET and MRI without compromising biologic specificity.

\section{CONCLUSION}

Emerging methods that exploit the intrinsic chemical and physical properties of nanoparticles have sparked a revolution in surface-based radiochemistry. Although nanoparticles remain inherently challenging systems, they present unparalleled opportunities for developing multimodality radiotracers. The expansion of chelate-free radiochemistry has broadened the tools available to radiochemists, narrowing the gap between academic curiosity and clinical translation. Future advancements will likely utilize intrinsically labeled nanomedicines as multimodality imaging agents that target specific disease biomarkers.

\section{DISCLOSURE}

Jason P. Holland received funding from the Swiss National Science Foundation (SNSF Professorship PP00P2_163683), the European Research Council (ERC-StG-2015, NanoSCAN-676904), and the University of Zurich. No other potential conflict of interest relevant to this article was reported.

\section{REFERENCES}

1. Wagner V, Dullaart A, Bock AK, Zweck A. The emerging nanomedicine landscape. Nat Biotechnol. 2006;24:1211-1217.

2. Welch MJ, Hawker CJ, Wooley KL. The advantages of nanoparticles for PET. J Nucl Med. 2009;50:1743-1746.

3. Liu Y, Welch MJ. Nanoparticles labeled with positron emitting nuclides: advantages, methods, and applications. Bioconjug Chem. 2012;23:671-682.

4. Xing Y, Zhao J, Conti PS, Chen K. Radiolabeled nanoparticles for multimodality tumor imaging. Theranostics. 2014;4:290-306.

5. Zhao J, Zhou M, Li C. Synthetic nanoparticles for delivery of radioisotopes and radiosensitizers in cancer therapy. Cancer Nanotechnol. 2016;7:9.

6. Rivera-Gil P, Jimenez de Aberasturi D, Wulf V, et al. The challenge to relate the physicochemical properties of colloidal nanoparticles to their cytotoxicity. Acc Chem Res. 2013;46:743-749.

7. Hong H, Chen F, Cai W. Pharmacokinetic issues of imaging with nanoparticles: focusing on carbon nanotubes and quantum dots. Mol Imaging Biol. 2013;15:507-520.

8. Lapi SE, Welch MJ. A historical perspective on the specific activity of radiopharmaceuticals: what have we learned in the 35 years of the ISRC? Nucl Med Biol. 2012;39:601-608

9. Schühle DT, Caravan P. Metal-Based MRI Contrast Agents. Vol 2. Charlestown, MA: Elsevier; 2013;901-932.

10. Zhao Y, Liang M, Li X, et al. Bioengineered magnetoferritin nanoprobes for single-dose nuclear-magnetic resonance tumor imaging. ACS Nano. 2016;10: 4184-4191.

11. Goel S, Chen F, Ehlerding EB, Cai W. Intrinsically radiolabeled nanoparticles: an emerging paradigm. Small. 2014;10:3825-3830.

12. Sun $X$, Cai W, Chen $X$. Positron emission tomography imaging using radiolabeled inorganic nanomaterials. Acc Chem Res. 2015;48:286-294.

13. Stockhofe K, Postema JM, Schieferstein H, Ross TL. Radiolabeling of nanoparticles and polymers for PET imaging. Pharmaceuticals (Basel). 2014;7:392-418.

14. Unak G, Ozkaya F, Ilker Medine E, et al. Gold nanoparticle probes: design and in vitro applications in cancer cell culture. Colloids Surf B Biointerfaces. 2012;90:217-226.

15. Guerrero S, Herance JR, Rojas S, et al. Synthesis and in vivo evaluation of the biodistribution of a ${ }^{18} \mathrm{~F}$-labeled conjugate gold-nanoparticle-peptide with potential biomedical application. Bioconjug Chem. 2012;23:399-408.

16. Zhu J, Chin J, Wängler C, Wängler B, Lennox RB, Schirrmacher R. Rapid ${ }^{18} \mathrm{~F}-$ labeling and loading of PEGylated gold nanoparticles for in vivo applications. Bioconjug Chem. 2014;25:1143-1150.

17. Rojas S, Gispert JD, Abad S, et al. In vivo biodistribution of amino-functionalized ceria nanoparticles in rats using positron emission tomography. Mol Pharm. 2012;9:3543-3550.

18. Torres Martin de Rosales R, Tavaré R, Paul RL, et al. Synthesis of ${ }^{64} \mathrm{Cu}^{\mathrm{II}}$ - bis (dithiocarbamatebisphosphonate) and its conjugation with superparamagnetic iron oxide nanoparticles: in vivo evaluation as dual-modality PET-MRI agent. Angew Chem Int Ed Engl. 2011;50:5509-5513.

19. Torres Martin de Rosales R, Tavaré R, Glaria A, Varma G, Protti A, Blower PJ. ${ }^{99 m}$ Tc-bisphosphonate-iron oxide nanoparticle conjugates for dual-modality biomedical imaging. Bioconjug Chem. 2011;22:455-465.

20. Gaynor D, Griffith DM. The prevalence of metal-based drugs as therapeutic or diagnostic agents: beyond platinum. Dalton Trans. 2012;41:13239.

21. Sandiford L, Phinikaridou A, Protti A, et al. Bisphosphonate-anchored pegylation and radiolabeling of superparamagnetic iron oxide: long-circulating nanoparticles for in vivo multimodal (T1 MRI-SPECT) imaging. ACS Nano. 2013;7:500-512.

22. Devaraj NK, Keliher EJ, Thurber GM, Nahrendorf M, Weissleder R. ${ }^{18} \mathrm{~F}$ labeled nanoparticles for in vivo PET-CT imaging. Bioconjug Chem. 2009;20:397-401.

23. Thorek DLJ, Ulmert D, Diop N-FM, et al. Non-invasive mapping of deep-tissue lymph nodes in live animals using a multimodal PET/MRI nanoparticle. Nat Commun. 2014;5:3097. 
24. Boros E, Bowen AM, Josephson L, Vasdev N, Holland JP. Chelate-free metal ion binding and heat-induced radiolabeling of iron oxide nanoparticles. Chem Sci. 2015;6:225-236.

25. Goel S, Chen F, Cai W. Synthesis and biomedical applications of copper sulfide nanoparticles: from sensors to theranostics. Small. 2014;10:631-645.

26. Zhou M, Zhang R, Huang M, et al. A chelator-free multifunctional $\left[{ }^{64} \mathrm{Cu}\right] \mathrm{CuS}$ nanoparticle platform for simultaneous micro-PET/CT imaging and photothermal ablation therapy. J Am Chem Soc. 2010;132:15351-15358.

27. Wong RM, Gilbert DA, Liu K, Louie AY. Rapid size-controlled synthesis of dextran-coated, ${ }^{64} \mathrm{Cu}$-doped iron oxide nanoparticles. ACS Nano. 2012;6:34613467.

28. Zhao Y, Sultan D, Detering L, et al. Copper-64-alloyed gold nanoparticles for cancer imaging: improved radiolabel stability and diagnostic accuracy. Angew Chem Int Ed Engl. 2014;53:156-159.

29. Sun $X$, Huang $X$, Yan $X$, et al. Chelator-free ${ }^{64} \mathrm{Cu}$-integrated gold nanomaterials for positron emission tomography imaging guided photothermal cancer therapy. ACS Nano. 2014;8:8438-8446.

30. Guo W, Sun $\mathrm{X}$, Jacobson $\mathrm{O}$, et al. Intrinsically radioactive $\left[{ }^{64} \mathrm{Cu}\right] \mathrm{CuInS} / \mathrm{ZnS}$ quantum dots for PET and optical imaging: improved radiochemical stability and controllable Cerenkov luminescence. ACS Nano. 2015;9:488-495.

31. Yang L, Sundaresan G, Sun M, et al. Intrinsically radiolabeled multifunctional cerium oxide nanoparticles for in vivo studies. J Mater Chem B. 2013;1:1421.

32. Pellico J, Ruiz-Cabello J, Saiz-Alía M, et al. Fast synthesis and bioconjugation of ${ }^{68} \mathrm{Ga}$ core-doped extremely small iron oxide nanoparticles for PET/MR imaging. Contrast Media Mol Imaging. 2016;11:203-210.

33. Sun M, Hoffman D, Sundaresan G, Yang L, Lamichhane N, Zweit J. Synthesis and characterization of intrinsically radiolabeled quantum dots for bimodal detection. Am J Nucl Med Mol Imaging. 2012;2:122-135.

34. Zeng J, Jia B, Qiao R, et al. In situ ${ }^{111}$ In-doping for achieving biocompatible and non-leachable ${ }^{111}$ In-labeled $\mathrm{Fe}_{3} \mathrm{O}_{4}$ nanoparticles. Chem Commun (Camb). 2014; 50:2170-2172.

35. Yang Y, Sun Y, Cao T, et al. Hydrothermal synthesis of $\mathrm{NaLuF}_{4}:{ }^{153} \mathrm{Sm}, \mathrm{Yb}, \mathrm{Tm}$ nanoparticles and their application in dual-modality upconversion luminescence and SPECT bioimaging. Biomaterials. 2013;34:774-783.

36. Wang Y, Liu Y, Luehmann H, et al. Radioluminescent gold nanocages with controlled radioactivity for real-time in vivo imaging. Nano Lett. 2013; 13:581-585.

37. Black KCL, Wang Y, Luehmann HP, et al. Radioactive ${ }^{198} \mathrm{Au}$-doped nanostructures with different shapes for in vivo analyses of their biodistribution, tumor uptake, and intratumoral distribution. ACS Nano. 2014;8:4385-4394.

38. Wu Z, Yang S, Wu W. Shape control of inorganic nanoparticles from solution. Nanoscale. 2016;8:1237-1259.

39. McNeil SE. Nanoparticle therapeutics: a personal perspective. Wiley Interdiscip Rev Nanomed Nanobiotechnol. 2009;1:264-271.

40. Nel AE, Mädler L, Velegol D, et al. Understanding biophysicochemical interactions at the nano-bio interface. Nat Mater. 2009;8:543-557.

41. Chen F, Ellison PA, Lewis CM, et al. Chelator-free synthesis of a dual-modality PET/MRI agent. Angew Chem Int Ed Engl. 2013;52:13319-13323.

42. Chakravarty R, Valdovinos HF, Chen F, et al. Intrinsically germanium-69 labeled iron oxide nanoparticle: synthesis and in vivo dual-modality PET/MR imaging. Adv Mater. 2014;26:5119-5123.

43. Liu T, Shi S, Liang C, et al. Iron oxide decorated MoS2 nanosheets with double PEGylation for chelator-free radiolabeling and multimodal imaging guided photothermal therapy. ACS Nano. 2015;9:950-960.

44. Goel S, Chen F, Luan S, et al. Engineering intrinsically zirconium-89 radiolabeled self-destructing mesoporous silica nanostructures for in vivo biodistribution and tumor targeting studies. Adv Sci (Weinh). 2016;3:1600122.

45. Ai F, Goel S, Zhan Y, et al. Intrinsically ${ }^{89} \mathrm{Zr}$-labeled $\mathrm{Gd}_{2} \mathrm{O}_{2} \mathrm{~S}$ :Eu nanophosphors with high in vivo stability for dual-modality imaging. Am J Transl Res. 2016; 8:5591-5600.
46. Shi S, Xu C, Yang K, et al. Chelator-free radiolabeling of nanographene: breaking the stereotype of chelation. Angew Chem Int Ed Engl. 2017;56:2889-2892.

47. Ellison PA, Chen F, Goel S, et al. Intrinsic and stable conjugation of thiolated mesoporous silica nanoparticles with radioarsenic. ACS Appl Mater Interfaces. 2017;9:6772-6781.

48. Chen F, Valdovinos HF, Hernandez R, Goel S, Barnhart TE, Cai W. Intrinsic radiolabeling of titanium-45 using mesoporous silica nanoparticles. Acta Pharmacol Sin. 2017;38:907-913.

49. Shaffer TM, Wall MA, Harmsen S, et al. Silica nanoparticles as substrates for chelator-free labeling of oxophilic radioisotopes. Nano Lett. 2015;15:864-868.

50. Pham TN, Lengkeek NA, Greguric I, et al. Tunable and noncytotoxic PET/ SPECT-MRI multimodality imaging probes using colloidally stable ligand-free superparamagnetic iron oxide nanoparticles. Int J Nanomedicine 2017;12:899909.

51. Wall MA, Shaffer TM, Harmsen S, et al. Chelator-free radiolabeling of SERRS nanoparticles for whole-body PET and intraoperative Raman imaging. Theranostics. 2017;7:3068-3077.

52. Radović M, Calatayud MP, Goya GF, et al. Preparation and in vivo evaluation of multifunctional ${ }^{90}$ Y-labeled magnetic nanoparticles designed for cancer therapy. J Biomed Mater Res A. 2015;103:126-134.

53. Abou DS, Thorek DLJ, Ramos NN, et al. ${ }^{89} \mathrm{Zr}$-labeled paramagnetic octreotideliposomes for PET-MR imaging of cancer. Pharm Res. 2013;30:878-888.

54. Cui X, Belo S, Krüger D, et al. Aluminium hydroxide stabilised $\mathrm{MnFe}_{2} \mathrm{O}_{4}$ and $\mathrm{Fe}_{3} \mathrm{O}_{4}$ nanoparticles as dual-modality contrasts agent for MRI and PET imaging. Biomaterials. 2014;35:5840-5846.

55. Sun Y, Yu M, Liang S, et al. Fluorine-18 labeled rare-earth nanoparticles for positron emission tomography (PET) imaging of sentinel lymph node. Biomaterials. 2011;32:2999-3007.

56. Cui X, Mathe D, Kovács N, et al. Synthesis, characterization, and application of core-shell $\mathrm{Co}_{0.16} \mathrm{Fe}_{2.84} \mathrm{O}_{4} @ \mathrm{NaYF}_{4}(\mathrm{Yb}, \mathrm{Er})$ and $\mathrm{Fe}_{3} \mathrm{O}_{4} @ \mathrm{NaYF}_{4}(\mathrm{Yb}, \mathrm{Tm})$ nanoparticle as trimodal (MRI, PET/SPECT, and optical) imaging agents. Bioconjug Chem. 2016;27:319-328.

57. Sharma R, Xu Y, Kim SW, et al. Carbon-11 radiolabeling of iron-oxide nanoparticles for dual-modality PET/MR imaging. Nanoscale. 2013;5:7476-7483.

58. Son DH, Hughes SM, Yin Y, Alivisatos AP. Cation exchange reactions in ionic nanocrystals. Science. 2004;306:1009-1012.

59. Sun X, Huang X, Guo J, et al. Self-illuminating ${ }^{64} \mathrm{Cu}$-doped $\mathrm{CdSe} / \mathrm{ZnS}$ nanocrystals for in vivo tumor imaging. J Am Chem Soc. 2014;136:1706-1709.

60. Sun Y, Zhu X, Peng J, Li F. Core-shell lanthanide upconversion nanophosphors as four-modal probes for tumor angiogenesis imaging. ACS Nano. 2013;7:11290-11300

61. Di Pasqua AJ, Yuan H, Chung Y, et al. Neutron-activatable holmium-containing mesoporous silica nanoparticles as a potential radionuclide therapeutic agent for ovarian cancer. J Nucl Med. 2013;54:111-116.

62. Munaweera I, Shi Y, Koneru B, et al. Chemoradiotherapeutic magnetic nanoparticles for targeted treatment of nonsmall cell lung cancer. Mol Pharm. 2015;12:3588-3596.

63. Pérez-Campaña C, Moya SE, Ziolo RF, et al. Biodistribution of different sized nanoparticles assessed by positron emission tomography: a general strategy for direct activation of metal. ACS Nano. 2013;7:3498-3505.

64. Pérez-Campaña C, Gómez-Vallejo V, Martin A, et al. Tracing nanoparticles in vivo: a new general synthesis of positron emitting metal oxide nanoparticles by proton beam activation. Analyst. 2012;137:4902-4906.

65. Boerman OC, Laverman P, Oyen WJG, Corstens FHM, Storm G. Radiolabeled liposomes for scintigraphic imaging. Prog Lipid Res. 2000;39:461-475.

66. Cisneros BT, Law JJ, Matson ML, Azhdarinia A, Sevick-Muraca EM, Wilson LJ. Stable confinement of positron emission tomography and magnetic resonance agents within carbon nanotubes for bimodal imaging. Nanomedicine. 2014;9:2499-2509. 